\title{
Video-assisted Thoracoscopic surgery (VATS) lobectomy for lung cancer does not induce a procoagulant state
}

Thomas Decker Christensen ${ }^{1,2^{*}}$ (D), Henrik Vad ${ }^{1,2}$, Søren Pedersen ${ }^{3,4}$, Peter B. Licht ${ }^{5}$, Mads Nybo ${ }^{6}$, Kåre Hornbech? Nora Elisabeth Zois ${ }^{8}$ and Anne-Mette Hvas ${ }^{9,10}$

\begin{abstract}
Background: Changes in the coagulation system in patients undergoing surgery for lung cancer have been sparsely investigated and the impact of the surgical trauma on the coagulation system is largely unknown in these patients. An increased knowledge could potentially improve the thromboprophylaxis regimes. The aim of this study was to assess the coagulation profile evoked in patients undergoing curative surgery by Video-Assisted Thoracoscopic Surgery (VATS) lobectomy for primary lung cancer.

Methods: Thirty-one patients diagnosed with primary lung cancer undergoing VATS lobectomy were prospectively included. The coagulation profile was assessed preoperatively and in the first two days postoperatively using a wide range of standard coagulation tests, dynamic whole blood coagulation measured by rotational thromboelastometry $\left(\mathrm{ROTEM}^{\circledast}\right)$ and thrombin generation evaluated by calibrated automated thrombography. Patients did not receive thromboprophylactic treatment. Data was analyzed using repeated measures one-way ANOVA.
\end{abstract}

Results: The standard coagulation parameters displayed only subtle changes after surgery and the ROTEM ${ }^{\oplus}$ and thrombin generation results remained largely unchanged.

Conclusions: Patients undergoing VATS lobectomy are normocoagulable in the preoperative state and a VATS lobectomy does not significantly influence the coagulation.

Trial registration: The trial is registered at ClinicalTrials.gov (Identifier: NCT01741506) and at EudraCTno. 2012-002409-23. Registered December 2012.

Keywords: Venous thrombosis, Blood coagulation test, Blood coagulation, Lung neoplasm, Thoracic surgery, Video assisted

\section{Background}

Cancer patients have an increased risk of venous thromboembolic events (VTE) consisting of deep venous thrombosis (DVT) or pulmonary embolism (PE) [1]. The risk factors for VTE are either patient-related (e.g. age, obesity), cancer-related (e.g. histopathologic type of cancer and advanced stage), treatment-related (e.g. surgical procedure, chemotherapy), or a combination of these factors $[2,3]$. Patients having potentially curative operations for lung

\footnotetext{
*Correspondence: tdc@clin.au.dk

'Department of Cardiothoracic and Vascular Surgery, Aarhus University Hospital, DK - 8200 Aarhus N, Denmark

${ }^{2}$ Department of Clinical Medicine, Aarhus University Hospital, DK - 8200

Aarhus N, Denmark

Full list of author information is available at the end of the article
}

cancer differ substantially from other lung cancer patients, since they suffer predominantly from early-stage disease. Accordingly, presence of a procoagulant imbalance is not as likely as it is in patients with more advanced disease $[3,4]$, but has not been investigated.

The influence on the coagulation system in patients undergoing thoracic (non-cardiac) surgery has only been sparsely investigated. Specifically, serial investigations of the coagulation system in the post-operative phase are lacking. Additional knowledge is warranted, since patients undergoing surgery for lung cancer still have an increased long-term risk of VTE [1-3].

New knowledge could potentially change the thromboprophylaxis regimen, e.g. regarding type of medication (Non-vitamin K antagonist oral anticoagulant (NOAC) 
versus Low-Molecular-Weight Heparin (LMWH)), dose, and timing (e.g. start-up after discharge from hospital and given on a long-term basis).

Papageorgiou et al. [5] found patients with lung adenocarcinoma undergoing lobectomy to be hypercoagulable pre- and postoperatively, whereas Swiniarska et al. [6] reported that surgery resulted in an activation of the coagulation system, however significantly more profound in pneumonectomies compared to lobectomies. In a porcine model Trabjerg et al. [7] showed that there was no significant activation of the coagulation system during lung surgery. Thus, results of the published studies are conflicting and inconclusive. In order to estimate the activation of the coagulation system, advanced and validated methods should be applied. Using rotational thromboelastometry $\left(\right.$ ROTEM $\left.^{\circ}\right)$, thrombin generation and standard coagulation parameters, a quantification of the coagulation system can be performed $[8,9]$.

We hypothesized that patients with primary lung cancer were hypercoagulable before surgery, and that the coagulation profile changes after surgery in terms of an activation of the coagulation system. The aim of this study was to assess the coagulation profile evoked in patients undergoing curative surgery by Video-Assisted Thoracoscopic Surgery (VATS) lobectomy for primary lung cancer.

\section{Methods}

\section{Patient population}

The present study is a sub-study to a larger randomized study already published, and the design is thus reported elsewhere [10].

Briefly, approximately 750 patients from a catchment area of 4,500,000 inhabitants are annually referred to Aarhus University Hospital, Rigshospitalet (University Hospital of Copenhagen) or Odense University Hospital, Denmark, to undergo surgery for primary lung cancer. From March 2013 to April 2015, patients who underwent lobectomy at one of these three sites were screened for eligibility.

Inclusion criteria were as follows: (1) Diagnosed with primary lung cancer with a preoperative stage IA-IB; (2) Surgery with expected lobectomy or bi-lobectomy using VATS; (3) Willingness to participate and ability to give informed oral and written consent; (4) No thromboprophylaxis received before or after surgery; and (5) > 18 years of age. Exclusion criteria were: (1) Thromboembolic event (either arterial or venous) within the past three months; (2) Pregnant or lactating; (3) Treatment with vitamin Kantagonist or a NOAC; or (4) Treatment with a platelet inhibitor if this was not paused for a minimum of 5 days (aspirin, clopidogrel or ticagrelor) or 7 days (prasugrel). No patients had received neoadjuvant chemo- and/or radiation therapy prior to surgery.

The patients were included after oral and written consent. The study protocol complied with the Helsinki II declaration and was approved by the local scientific ethical committee (File number: 1-10-72-364-12) and The Danish Data Protection Agency. The study was conducted according to Good Clinical Practice (GCP) standards and was monitored and approved by the GCP-unit, Aarhus University Hospital, Aarhus, Denmark. The trial is registered at ClinicalTrials.gov (Identifier: NCT01741506) and at EudraCTno. 2012-002409-23.

Included patients were randomized to treatment with either LMWH or no intervention. In the present study, only the subpopulation of patients receiving no intervention was included.

\section{Intervention}

All operations were performed in general anaesthesia with propofol and fentanyl. The VATS approach used has previously been described in details [11]. Briefly, an anterior approach with one incision and two port assist incisions were performed, and one chest tube was placed. All patients were intubated with a Carlens double-lumen tube, extubated immediately after surgery and stayed approximately $12 \mathrm{~h}$ in the high dependency unit and then transferred back to the ward. The patients were mobilized within hours of finishing the operation and no mechanical thromboprophylaxis was provided.

\section{Observation period and blood analyses}

Due to logistics it was not possible to perform the coagulation analyses during weekends; accordingly, only patients operated on Mondays, Tuesdays and Wednesdays were included. Accordingly, the inclusion period was somewhat elongated.

Blood samples were obtained and analyzed at the following three time-points: 1) Preoperatively; the day before surgery; 2) Postoperatively 0800 AM at day 1 ; and 3 ) Postoperatively $0800 \mathrm{AM}$ at day 2 .

The first $2 \mathrm{ml}$ of blood was discarded before drawing blood into tubes containing sodium citrate for ROTEM ${ }^{\circ}$ analyses, thrombin generation and standard coagulation analyses including: Activated partial thromboplastin time (APTT), International Normalized Ratio (INR), fibrinogen (functional), fibrin d-dimer, platelet count and coagulation factor VIII:Clot. Blood for ROTEM ${ }^{\circ}$ analyses were left at room temperature for $30 \mathrm{~min}$ before processing, whereas the remaining analyses were done either immediately as routine analyses or blood samples were centrifuged at $2800 \mathrm{~g}$ for $25 \mathrm{~min}$ and plasma was stored in aliquots at $-80{ }^{\circ} \mathrm{C}$ until analysis.

Regarding ROTEM ${ }^{\circ}$ (Tem International GmbH, Munich, Germany), three standard assays were performed: INTEM, EXTEM, and FIBTEM. We obtained the dynamic parameters of clot initiation (clotting time: CT, seconds (s)) and clot propagation (maximum velocity of clot formation: MaxVel, $\mathrm{mm} \times 100 / \mathrm{s}$, time to maximum velocity: tMaxVel, s). Whole 
blood clot strength was assessed by maximum clot firmness (MCF, $\mathrm{mm} \times 100)$.

Thrombin generation was evaluated by calibrated automated thrombograms (CAT; Thrombinoscope BV, Maastricht, the Netherlands) using platelet-poor plasma. The following parameters were analyzed: Lag-time until initial thrombin generation (minutes), maximum concentration of thrombin (peak, nM), time to peak (ttpeak, minutes), and the endogenous thrombin potential (ETP, $\mathrm{nM} \times \mathrm{x}$ minutes).

The normal range for the ROTEM $^{\circledR}$ was calculated based on data obtained from 73 healthy individuals previously published [12], and the normal range for thrombin generation were obtained from 90 individuals published by Vibede et al. [13].

APTT (Platelin LS, Organon, Munich, Germany), INR (Owren's PT-reagent, MediRox), fibrinogen (Clauss method, Siemens Dade reagent) were analyzed employing the CS2100i (Sysmex, Kobe, Japan) Coagulation factor VIII:Clot (factor VIII:Clot) was analyzed by ACL-TOP (Instrumental Laboratory, Bedford, MA, USA).

Preoperatively, the following baseline analyses were performed: haemoglobin, leukocytes, platelet count, creatinine, INR and C-reactive protein (CRP).

Preoperative (baseline) data in terms of clinical characteristics was collected systematically from medical records. Furthermore, intra- and postoperative data (operating time, bleeding during surgery, transfusion, total drain loss, VTE and adverse events, length of stay and pathological staging) were registered prospectively in a case report form.

\section{Statistical analyses, endpoints and sample size}

Baseline and postoperative characteristics were tested for normal distribution and hence presented as either mean and standard deviation (SD) or median and 95\% confidence interval $(\mathrm{CI})$ or as minimum to maximum values.

The results of the coagulation analyses were tested using repeated measures one-way ANOVA, sphericity was not assumed and alpha was set to 0.05. All three measurements (preoperatively, 1. postoperative day and 2. postoperative day) were needed, otherwise the patient's measurements for that specific parameter was excluded.

Microsoft $^{\oplus}$ Excel $^{\oplus}$ for Mac 2011 (Microsoft ${ }^{\oplus}$, Seattle, USA) and GraphPad Prism 6 for Mac (GraphPad Software, Inc., CA, USA) were used for the statistical analyses.

The study was explorative in nature, and a sample size calculation was therefore not performed prior to the inclusion of patients.

\section{Results}

Figure 1 displays the trial flowchart. A total of 81 VATSpatients were randomized; 40 patients to the LMWH arm and 41 patients to the no intervention arm (a trial flowchart is displayed in Fig. 1). Patients randomized to the LMWH arm were excluded in the present study, and these results are published elsewhere [10].

In the no intervention arm, 10 patients were excluded (Fig. 1), and a total of 31 patients were therefore included in the present study. Among these, three patients had been given NSAID, and additionally three patients were converted to an open procedure. Three patients were operated at Odense University Hospital, three patients at Rigshospitalet (University Hospital of Copenhagen), and 25 at Aarhus University Hospital.

None of the included patients had a previously event of VTE.

Six patients (19\%) received aspirin before being admitted to surgery, but all patients paused their aspirin at least 5 days prior to surgery.

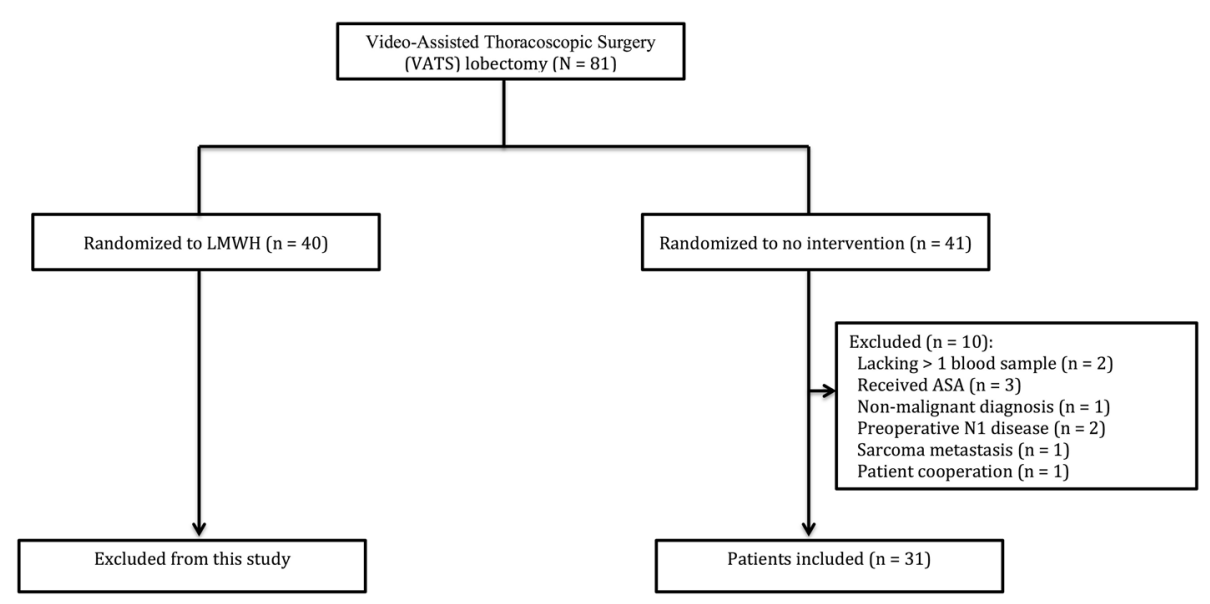

Fig. 1 Trial flowchart for patients planned for Video-Assisted Thoracoscopic Surgery (VATS) lobectomy for primary lung cancer. The number of patients assessed for eligibility is not shown. Abbreviations: n/N: numbers; ASA: Acetylsalicylic acid (aspirin); LMWH: Low-Molecular-Weight Heparin; NSAID: Non Steroid Anti Inflammatory Drug 
Table 1 shows preoperative characteristics, while the intra- and postoperative data are displayed in Table 2. Three patients received transfusion during surgery; one patient received red blood cells, platelets and fresh frozen plasma; one patient received red blood cells and platelets and one patient received merely red blood cells.

The results of the ANOVA analyses regarding the standard coagulation blood tests, ROTEM $^{\circ}$ results and thrombin generation are displayed graphically in Figs. 2, 3, and 4.

The findings illustrated in Figs. 2, 3, 4 shows a statistically significant change ( $p$-values are displayed in Figs. 2, 3 , and 4 ) over time from pre- to the postoperatively state for a total of nine parameters. An increased fibrinogen, fibrin d-dimer, factor VIII:Clot and FIBTEM MCF was found reflecting a hypercoagulable state The following changes reflected a hypocoagulable state: an increase in

Table 1 Baseline data (preoperative) of the study population prior to Video-Assisted Thoracoscopic Surgery (VATS) lobectomy for lung cancer, $n=31$

\begin{tabular}{|c|c|}
\hline Variables & Values \\
\hline Age (years) & $67(10)$ \\
\hline Sex (female/male), n & 20/11 \\
\hline Non smoker/ex-smoker/ active smoker, n & $1 / 19 / 11$ \\
\hline Pack years of smoking & $30(20)$ \\
\hline FEV 1 (\% of expected) & $91(20)$ \\
\hline DLCO (\% of expected) & $69(17)$ \\
\hline BMl & $25(4)$ \\
\hline \multicolumn{2}{|l|}{ Co-morbidity ${ }^{a}, \mathrm{n}(\%)$ : } \\
\hline Diabetes mellitus & $2(7)$ \\
\hline Hypertension & $12(39)$ \\
\hline Hyperlipidemia & $10(31)$ \\
\hline Cardiac and/or vascular disease & $4(13)$ \\
\hline Previous malignant disease & $8(26)$ \\
\hline ASA prescribed, $\mathrm{n}(\%)$ & $6(19)$ \\
\hline \multicolumn{2}{|l|}{ Laboratory analyses (reference interval): } \\
\hline $\begin{array}{l}\text { B - Haemoglobin (women: } 7.3-9.5 \mathrm{mmol} / \mathrm{L} \text {; men: } \\
8.3-10.5 \mathrm{mmol} / \mathrm{L} \text { ) }\end{array}$ & $8.5(0.7)$ \\
\hline B - Leukocytes $\left(3.5-10.0 \times 10^{9} / \mathrm{L}\right)$ & $7.5(2.0)$ \\
\hline $\begin{array}{l}P \text { - Creatinine (women: } 45-90 \mu \mathrm{mol} / \mathrm{L} \text {; men: } \\
60-105 \mu \mathrm{mol} / \mathrm{L} \text { ) }\end{array}$ & $72(18)$ \\
\hline B - Platelet count $\left(145-400 \times 10^{9} / \mathrm{L}\right)$ & $287(85)$ \\
\hline$P$ - C-reactive protein $(8 \mathrm{mg} / \mathrm{L})$ & $4(4)$ \\
\hline$P-\operatorname{INR}(<1.2)$ & $1.0(0.1)$ \\
\hline P - APTT (25-38 s) & $30(4)$ \\
\hline
\end{tabular}

Abbreviations: $B$ Blood, $N / n$ Numbers, $P$ Plasma, APTT Activated partial thromboplastin time, ASA Acetylsalicylic acid (aspirin), BMI Body Mass Index, DLCO Diffusion Capacity of the Lung for Carbon Monoxide, FEV1 Forced Expiratory Volume in one second, INR International Normalized Ratio All values are provided either as mean and (standard deviation) or numbers and (percentage)

${ }^{a}$ Defined as the patient being in medical treatment for the disease
Table 2 Peri- and postoperative data of the study population following Video-Assisted Thoracoscopic Surgery (VATS) lobectomy for lung cancer, $n=31$

\begin{tabular}{|c|c|}
\hline Variables (n) & Values \\
\hline \multicolumn{2}{|l|}{ Type of lobectomy (n) } \\
\hline Right Upper Lobe & 10 \\
\hline Right Middle Lobe & 1 \\
\hline Right Lower Lobe & 10 \\
\hline Left Upper Lobe & 7 \\
\hline Left Lower Lobe & 3 \\
\hline Operating time (h:m) & $2: 31(0: 47)$ \\
\hline Bleeding/drainage during surgery (ml) & $100(0-2000)$ \\
\hline Use of inotropes (patients, n) ${ }^{a}$ & 15 \\
\hline Re-operated & 1 \\
\hline Total amount of fluid in the chest drain (ml) & $850(50-6135)$ \\
\hline Complications $^{\mathrm{b}}$ & $5^{c}$ \\
\hline VTE events & 0 \\
\hline Death & 0 \\
\hline Total length of stay (days) & $5.5(3.2-20.0)$ \\
\hline \multicolumn{2}{|l|}{ Type of cancer } \\
\hline Adenocarcinoma & 23 \\
\hline Squamous cell carcinoma & 4 \\
\hline Carcinoid (all types) & 1 \\
\hline Others $^{d}$ & 3 \\
\hline \multicolumn{2}{|l|}{ Pathological staging } \\
\hline Stage IA + B & 27 \\
\hline Stage $\| A+B$ & 4 \\
\hline Microscopically free resection margins (R0) & 31 \\
\hline
\end{tabular}

Abbreviations: $H$ Hours, $N / n$ Numbers, $M$ Minutes, $m /$ milliliter, VTE Venous Thromboembolic Events

Operating time was normal distributed and is shown as mean and (standard deviation) in parenthesis, but the other data were not normally distributed and are accordingly displayed as median and (minimum to maximum) aPredominantly small doses of methaoxidrin or efedrin

bincludes myocardial infarction, apoplexia cerebri and atrial fibrillation

${ }^{c}$ Apoplexia cerebri $(n=2)$ and atrial fibrillation $(n=3)$

dincludes small cell carcinoma, neuroendocrine and sarcomatoid tumor

INR, and a decrease in platelet count, INTEM tMaxVel and time to peak.

\section{Discussion}

The main findings of the present study were that patients undergoing VATS lobectomy are normocoagulable in the preoperative state and that VATS lobectomy does not have significantly impact on coagulation. Only very few variables were found statistically significantly affected on the postoperative day 2 . However, none of these changes was of clinical relevance.

This is a sub-study of a larger study, where patients undergoing curative surgery for primary lung cancer with the use of VATS were randomized to either LMWH or no 

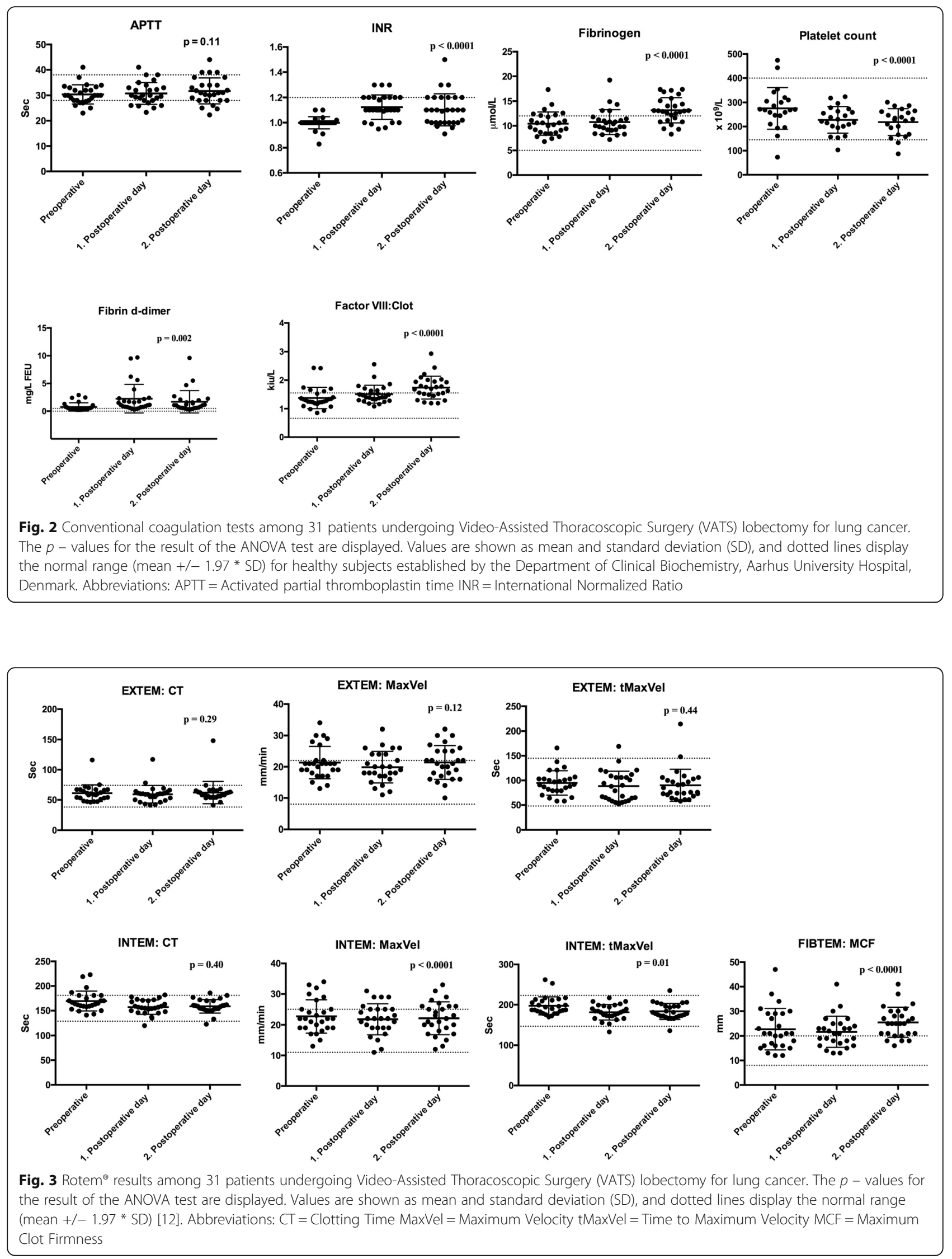

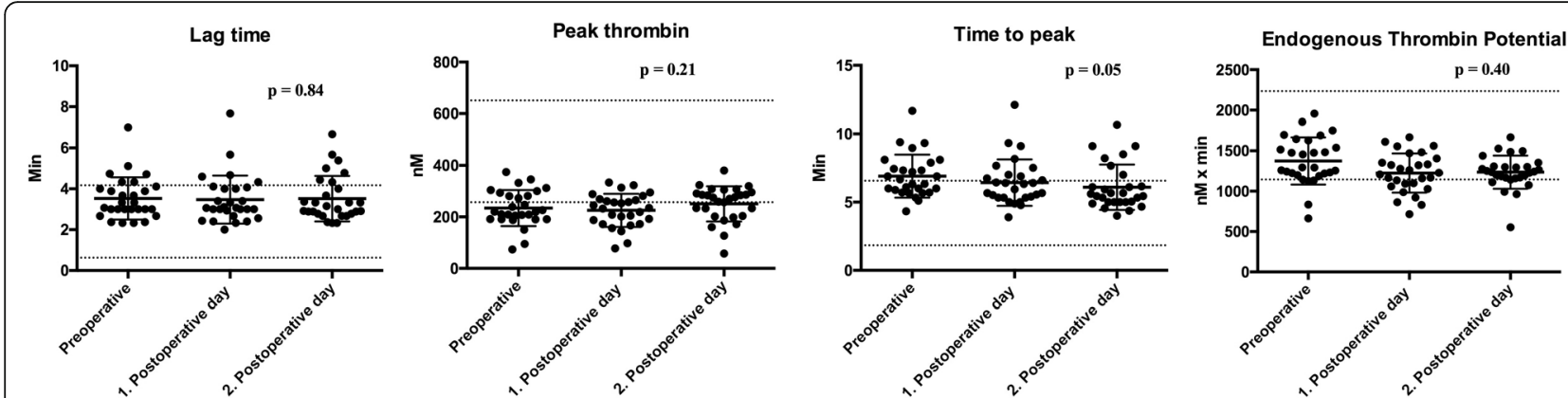

Fig. 4 Thrombin generation among 31 patients undergoing Video-Assisted Thoracoscopic Surgery (VATS) lobectomy for lung cancer. The $p$ - values for the result of the ANOVA test are displayed. Values are shown as mean and standard deviation (SD), and dotted lines display the normal range (mean +/- 1.97* SD) [13]

thromboprophylaxis [10]. In that study, we found that the use of LMWH administered once daily as thromboprophylaxis did not alter the coagulation profile per se.

In order to investigate the temporal changes in the coagulation profiles of surgical patients, we included a group of lung cancer patients undergoing surgery receiving no thromboprophylaxis. Accordingly, we were able to follow the natural course of the coagulation system pre- and postoperatively.

The previously published studies within this area have some major limitations. The study by Trabjerg et al. [7] employed a porcine non-cancerous model, which does not necessarily reflect changes occurring in human coagulation [14]. The studies by Papageorgiou et al. [5] and Swiniarska et al. [6] had the drawback of using mainly standard blood analysis and determination of tissue factor concentration instead of global analysis as e.g. ROTEM ${ }^{\circ}$ and thrombin generation. In contrast, the parameters used in the present study reflected the overall impact on the coagulation system [8, 9]. Moreover, it has even been advocated that thromboelastometry can be able to predict the risk of VTE after surgery [15].

The changes in the parameters fibrin d-dimer, factor VIII:Clot and FIBTEM MCF reflected a subtle hypercoagulability, while a small degree of hypocoagulability was displayed in the parameters INR, platelet count, INTEM, tMaxVel and time to peak. The absolute changes in the coagulation parameters were however small indicating that these changes are probably not of major clinical relevance.

Our patients were operated by a minimal invasive approach and VATS lobectomies are supposed to induce a reduced surgical stress compared to an open procedure [11], which probably adds to the low activation of and impact on the coagulation system. Accordingly, one can question whether this patient group benefits from thromboprophylaxis provided in the period around surgery. Further studies are obviously needed including clinical endpoints.
We chose not to include analyses of coagulation parameters during surgery, since they are influenced by several intra-operative factors such as anesthesia, surgical stress and bleeding, which introduce considerable variability. Furthermore, our aim was to investigate the changes in the coagulation profile evoked by surgery.

Of note, the present study was merely a descriptive study, since we did not perform any interventions.

Our study has some limitations because we used surrogate and not clinical endpoints in terms of VTE and bleeding events. However, the use of clinical endpoints would imply inclusion of a very large group of patients, because these events are relatively rare. Additionally, we only included blood samples up to two days after surgery and in a limited number of patients $(n=31)$.

The relatively low number of patients limits the conclusions that can be drawn from this study.

Nevertheless, we find that this fully reflects the entire intra- and postoperative period, and blood samples drawn e.g. on postoperative day 14 and day 30 would probably not have altered our conclusion, because the investigated parameters were un-altered following surgery. A larger group of patients would of course have solidified our conclusions.

We found a relatively marked variability between the individual patients at different time points for some of the ROTEM $^{\circ}$ parameters and thrombin generation variables. Even among healthy individuals the measurements in question have a relatively large variability and this fact should be taken into consideration when interpreting the results.

Besides coagulation, it would be relevant to investigate inflammatory markers in order to elucidate the crosstalk between the inflammatory and the coagulation systems. However, this was beyond the scope of this study.

Changes in coagulation are predominantly reported in adenocarcinomas, but is likely to also exist in other histopathological types [6]. However, we could have obtained more clearcut results, if we had included merely one type of cancers i.e. adenocarcinomas. Yet, the majority of cases 
included herein were adenocarcinomas (23 out of 31 patients corresponding to $74 \%$ ).

We had a relatively high dropout rate due to the complex set-up with the blood sampling and analyses, potential interaction with medication (e.g. aspirin and NSAID) and accordingly a slow inclusion rate of patients. However, this is not assumed to have interfered with either the internal or external validity of our study.

Since all patients paused their aspirin at least 5 days prior to surgery, it is thus unlikely that aspirin administration had any impact on the conclusions drawn from this study.

The surgical procedure and technique remained the same over the entire study period. Three patients were converted to an open procedure, mainly due to bleeding, and three patients received NSAID postoperatively. We decided to include these patients in our analysis, and the inclusion did not affect the overall conclusions drawn from the study.

In order to perform a repeated measures ANOVA analyses, no missing observations were tolerated. Hence, a smaller number of patients were excluded from the statistical analysis of the respective parameters resulting in a resultant sample size of 26-29.

\section{Conclusions}

Patients undergoing VATS lobectomy are normocoagulable in the preoperative state and a VATS lobectomy does not significantly influence the coagulation.

\begin{abstract}
Abbreviations
APTT: Activated partial thromboplastin time; CAT: Calibrated automated thrombograms; Cl: Confidence interval; CRP: C-reactive protein; DVT: Deep venous thrombosis; GCP: Good Clinical Practice; INR: International Normalized Ratio; LMWH: Low-Molecular-Weight Heparin; MaxVel: maximum velocity of clot formation; MCF: Maximum clot firmness; NOAC: Non-vitamin K antagonist oral anticoagulant; NSAID: Non Steroid Anti Inflammatory Drug; PE: Pulmonary embolism; SD: Standard deviation; tMaxVel: Time to maximum velocity; VATS: Video-Assisted Thoracoscopic Surgery (VATS); VTE: Venous thromboembolic events
\end{abstract}

\section{Acknowledgements}

We wish to thank research nurse Vibeke Laursen for helping and coordinating all of the practical issues of conducting this study and laboratory technician Mai Stenulm Therkelsen and Vivi Bo Mogensen for blood sampling and analysis. Hans Pilegaard, MD, and Professor Vibeke E. Hjortdal are thanked for making the study practically feasible.

\section{Funding}

The study was financially supported by Arvid Nilssons fond, Snedkermester Sophus Jacobsen og hustru Astrid Jacobsens Fond and Fru Agnes Niebuhr Andersons Cancerforskningsfonds pris. The work was done independent of the funders.

\section{Availability of data and materials}

Data are saved into an appropriately designed Excel spreadsheet and Prism statistical program. The data sets used and analyzed are available from the corresponding author on request.

\section{Authors' contributions}

Contributions: TDC, HV, PBL, MN and $\mathrm{AMH}$ conceived and designed the study; TDC, HV, SP, PBL, MN, KH, NZ and AMH collected and assembled the data; TDC and $\mathrm{AMH}$ analyzed and interpreted the data; and all authors wrote and provided final approval of the manuscript.

\section{Ethics approval and consent to participate}

The patients were included after oral and written consent. The study protocol complied with the Helsinki II declaration and was approved by the local scientific ethical committee (File number: 1-10-72-364-12) and The Danish Data Protection Agency. The study was conducted according to Good Clinical Practice (GCP) standards and was monitored and approved by the GCP-unit, Aarhus University Hospital, Aarhus, Denmark. The trial is registered at ClinicalTrials.gov (Identifier: NCT01741506) and at EudraCTno. 2012-002409-23.

\section{Consent for publication}

Not applicable

\section{Competing interests}

Thomas Decker Christensen has been on the speaker bureaus for AstraZeneca, Boehringer-Ingelheim, Pfizer, Roche Diagnostics, Takeda, BristolMyers Squibb and Merck Sharp \& Dohme (MSD) and has been in an Advisory Board for Bayer, Merck Sharp \& Dohme (MSD) and Boehringer-Ingelheim. Anne-Mette Hvas has received speaker's fees from CSL Behring, Bayer, Boehringer-Ingelheim, Bristol-Myers Squibb and Leo Pharma and unrestricted research support from Octapharma, CSL Behring and Leo Pharma. Mads Nybo has received speaker's fees from Astra Zeneca, Pfizer, BoehringerIngelheim, and Roche Diagnostics. Peter Licht has received speaker's fees from Ethicon Endo-Surgery. Other authors - none declared.

\section{Publisher's Note}

Springer Nature remains neutral with regard to jurisdictional claims in published maps and institutional affiliations.

\section{Author details}

${ }^{1}$ Department of Cardiothoracic and Vascular Surgery, Aarhus University Hospital, DK - 8200 Aarhus N, Denmark. ²Department of Clinical Medicine, Aarhus University Hospital, DK - 8200 Aarhus N, Denmark. ${ }^{3}$ Department of Anesthesiology and Intensive Care, Aarhus University Hospital, DK - 8200 Aarhus N, Denmark. ${ }^{4}$ Department of Clinical Medicine, Aarhus University Hospital, DK - 8200 Aarhus N, Denmark. ${ }^{5}$ Department of Cardiothoracic and Vascular Surgery, Odense University Hospital, DK - 5000 Odense C, Denmark. ${ }^{6}$ Department of Clinical Biochemistry, Odense University Hospital, DK - 5000 Odense C, Denmark. 'D Department of Cardio-thoracic Surgery, Rigshospitalet, Copenhagen University Hospital, DK - 2100 Copenhagen, Denmark.

${ }^{8}$ Department of Clinical Biochemistry, Rigshospitalet, Copenhagen University Hospital, DK - 2100 Copenhagen, Denmark. ${ }^{9}$ Department of Clinical Biochemistry, Aarhus University Hospital, DK - 8200 Aarhus N, Denmark.

${ }^{10}$ Department of Clinical Medicine, Aarhus University Hospital, DK - 8200 Aarhus N, Denmark.

Received: 15 September 2017 Accepted: 11 December 2017 Published online: 20 December 2017

References

1. Akl EA, van Doormaal FF, Barba M, et al. Parenteral anticoagulation may prolong the survival of patients with limited small cell lung cancer: a Cochrane systematic review. J Exp Clin Cancer Res. 2008;27:4.

2. Corrales-Rodriguez $L$, Blais N. Lung cancer associated venous thromboembolic disease: a comprehensive review. Lung Cancer. 2012;75:1-8.

3. Kyriazi $\mathrm{V}$, Theodoulou $\mathrm{E}$. Assessing the risk and prognosis of thrombotic complications in cancer patients. Arch Pathol Lab Med. 2013;137:1286-95.

4. Attaran S, Somov P, Awad WI. Randomised high- and low-dose heparin prophylaxis in patients undergoing thoracotomy for benign and malignant disease: effect on thrombo-elastography. Eur J Cardiothorac Surg. 2010;37: 1384-90.

5. Papageorgiou C, Vandreden P, Marret E, et al. Lobectomy and postoperative thromboprophylaxis with enoxaparin improve blood hypercoagulability in patients with localized primary lung adenocarcinoma. Thromb Res. 2013; 132:584-91.

6. Świniarska J, Zekanowska E, Dancewicz M, Bella M, Szczesny TJ, Kowalewski J. Pneumonectomy due to lung cancer results in a more pronounced activation of coagulation system than lobectomy. Eur J Cardio-Thoracic Surg. 2009;36:1064-8.

7. Trabjerg TB, Sander KD, Nybo M, Licht PB. Coagulation and fibrinolysis during lung surgery:an experimental study. Interact Cardiovasc Thorac Surg. 2014;19:567-71. 
8. Papa ML, Capasso F, Pudore L, et al. Thromboelastographic profiles as a tool for thrombotic risk in digestive tract cancer. Exp Oncol. 2007;29:111-5.

9. Sørensen B, Johansen P, Christiansen K, Woelke M, Ingerslev J. Whole blood coagulation thrombelastographic profiles employing minimal tissue factor activation. J Thromb Haemost. 2003;1:551-8.

10. Christensen TD, Vad H, Pedersen S, et al. Coagulation profile in patients undergoing video-assisted thoracoscopic lobectomy: a randomized, controlled trial. PLoS One. 2017;12:e0171809.

11. Laursen LØ, Petersen RH, Hansen HJ, Jensen TK, Ravn J, Konge L. Videoassisted thoracoscopic surgery lobectomy for lung cancer is associated with a lower 30-day morbidity compared with lobectomy by thoracotomy. Eur J Cardiothorac Surg. 2016;49:870-5.

12. Andersen MG, Hvas CL, Tønnesen E, Hvas AM. Thromboelastometry as a supplementary tool for evaluation of hemostasis in severe sepsis and septic shock. Acta Anaesthesiol Scand. 2014:58:525-33.

13. Vibede E, Hvas CL, Tønnesen E, Hvas AM. The effect of fresh frozen plasma in critically ill patients. Acta Anaesthesiologica Scandinavia. 2017;61:492-501.

14. Kessler U, Grau T, Gronchi F, et al. Comparison of porcine and human coagulation by thrombelastometry. Thromb Res. 2011;128:477-82.

15. Hincker A, Feit J, Sladen RN, Wagener G. Rotational thromboelastometry predicts thromboembolic complications after major non-cardiac surgery. Crit Care. 2014;18:549.

\section{Submit your next manuscript to BioMed Central} and we will help you at every step:

- We accept pre-submission inquiries

- Our selector tool helps you to find the most relevant journal

- We provide round the clock customer support

- Convenient online submission

- Thorough peer review

- Inclusion in PubMed and all major indexing services

- Maximum visibility for your research

Submit your manuscript at www.biomedcentral.com/submit 